\title{
Calcium antagonists, nitrates, and new antianginal drugs
}

\author{
DEREK MACLEAN，JOHN FEELY
}

Considerable progress in the medical as well as the surgical management of angina has been made in the past decade. An appreciation of the pharmacokinetics of the "oldest" antianginal drugs, the nitrates, has lead to the development of effective long acting preparations and novel techniques of administration. On the other hand, calcium antagonists represent a new approach to the treatment of angina as an alternative to the now well established beta-blockers, and the two are often complementary.

We now have a greater understanding of how to reduce work done by the heart (hence oxygen consumption) and thus relieve angina. This may be achieved in various ways (fig 1) such as by reducing venous return (preload) with nitrates or by reducing heart rate (beta-blockers) or muscle contractility (beta-blockers, calcium antagonists) directly. The resistance against which the

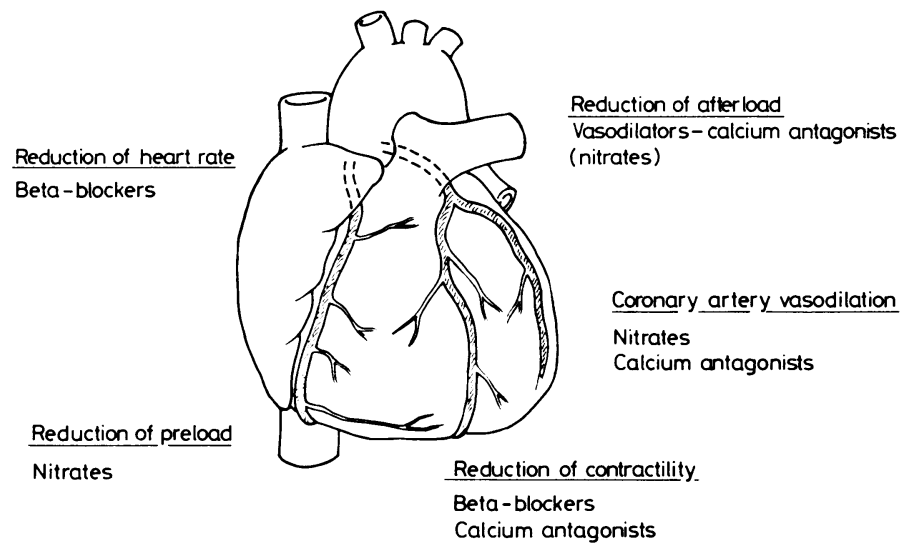

FIG 1 -Site of action of antianginal drugs.

heart has to pump (afterload) may be reduced by arterial dilatation (calcium antagonists). Alternatively, the oxygen supply to the heart may be increased by reducing spasm of the coronary arteries (calcium antagonists) or possibly redistributing blood flow to ischaemic areas (nitrates). Clearly, drugs may act by various different mechanisms and may also be successfully used in combination with glyceryl trinitrate in angina and with long acting nitrates, beta-blockers, or calcium antagonists (or combinations thereof) to prevent angina. The use of vasodilators in heart failure and of beta-blockers in angina are considered in separate articles in this series.

Department of Pharmacology and Therapeutics, Ninewells Hospital and Medical School, Dundee DD1 9SY

DEREK MACLEAN, PHD, FRCP, senior lecturer

JOHN FEELY, MD, MRCPI, lecturer

Correspondence to: Dr J Feely.

\section{Calcium antagonists}

Calcium channel blocking agents or antagonists are an impor- के tant new class of drug that reduce myocardial work both directly $\vec{\circ}$ and in many cases also indirectly by causing vasodilatation. They are particularly useful in angina, being, along with the nitrates, the treatment of choice for coronary artery spasm. Their use in hypertension is also increasing. In addition, one of the group, verapamil, is highly effective in managing supraventricular and junctional tachycardias.

To appreciate fully the differing profile of activity of the members of the group an understanding of their mode of action is necessary. In the myocardium and vascular smooth muscle an essential step in the process of contraction is the entry of calcium ions into the cells. In heart muscle this occurs during the "slow current" or plateau phase (2) of the action potential (fig 2).

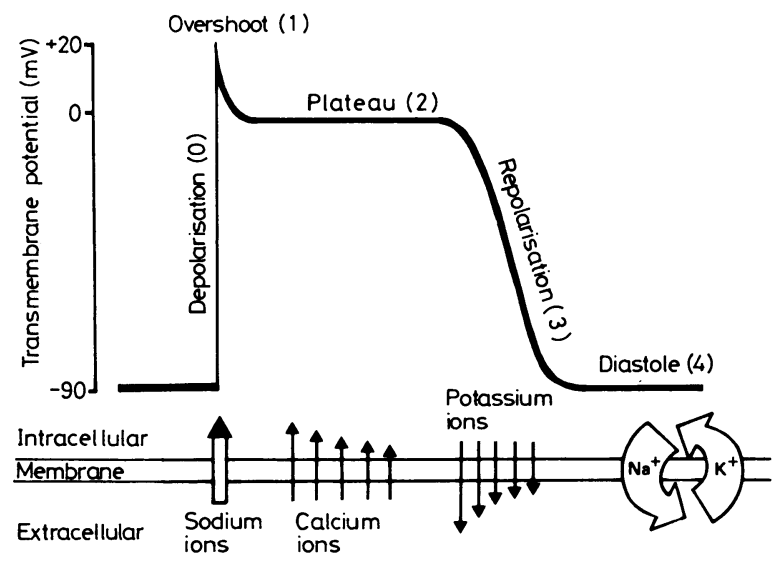

FIG 2-Schematic summary of the generation and different phases of an action potential in cardiac cells.

This entry of calcium ions into the cells causes the contractile protein to interact, thus leading to fibre shortening and increasing myocardial wall tension. The degree of this contractility (or positive inotropic state) is regulated by the amount of calcium ions that reach the contractile proteins, both from outside the cells but also from "activator" calcium ions released from an intracellular calcium pool during the action potential. The fibres of the atrioventricular node and contraction in the arterial and venous smooth muscle cells, unlike contraction in heart muscle, rely predominantly on the entry of external calcium ions. Calcium antagonists effectively block the entry of calcium ions through the slow calcium channels ("slow channel blockers"). They thereby reduce the strength of cardiac muscle contraction, slow conduction through the atrioventricular node with a consequent increase in the $P-R$ interval on the electrocardiogram, and also decrease arterial and venous smooth muscle tone.

Numerous calcium antagonists have been developed, and many are now available in the United Kingdom (table), with 
nifedipine and verapamil being the most widely used. Each differs considerably from the others, however, both in terms of potency and in their differential effects on heart muscle contraction, atrioventricular node conduction, and arterial and venous smooth muscle tone. Some, such as verapamil and perhexiline, have additional modes of action. Nifedipine exerts its greatest effect on vascular smooth muscle, including that in the coronary arteries, but has much less effect on myocardial contractility and little influence on atrioventricular node conduction.

Comparison of calcium antagonists

\begin{tabular}{|c|c|c|c|c|}
\hline Drug (proprietary name) & $\begin{array}{c}\text { Negative } \\
\text { inotropic } \\
\text { effect }\end{array}$ & $\begin{array}{c}\text { Decreased } \\
\text { atrioventricular } \\
\text { node } \\
\text { conduction }\end{array}$ & $\begin{array}{l}\text { Decreased } \\
\text { smooth } \\
\text { muscle } \\
\text { tone }\end{array}$ & Oral dose range \\
\hline Nifedipine (Adalat) & + & - & +++ & $10-20 \mathrm{mg}$ three \\
\hline Verapamil (Cordilox) & ++ & ++ & ++ & $40-120 \mathrm{mg}$ three \\
\hline Lidoflazine (Clinium) & - & + & + & $\begin{array}{l}\text { times daly } \\
\text { increasing at } \\
\text { weekly intervals } \\
\text { to twice or three } \\
\text { times daily } \\
\text { (maximum) }\end{array}$ \\
\hline Prenylamine (Synadrin) & + & + & ++ & $\begin{array}{l}60 \mathrm{mg} \text { three times } \\
\text { daily increasing to } \\
\text { five times daily }\end{array}$ \\
\hline Perhexiline (Pexid) & - & + & + & $\begin{array}{l}100-200 \mathrm{mg} \text { twice } \\
\text { daily }\end{array}$ \\
\hline
\end{tabular}

Verapamil, on the other hand, exerts a much greater effect than nifedipine on both myocardial contractility and atrioventricular node conduction as well as having a useful effect in reducing vascular smooth muscle tone. The reasons for the differential tissue specificity are not fully clear but are of considerable therapeutic importance.

\section{NIFEDIPINE}

Nifedipine is almost completely absorbed. It is detectable in blood, and a hypotensive effect is seen within five minutes of buccal or 20 minutes of oral administration. Peak concentrations occur one to two hours after o-al use, and the drug is about $90 \%$ bound to plasma proteins. It is metabolised in the liver to inactive metabolites that are primarily excreted by the kidney. Although the plasma half life is only four to five hours, the duration of effect may extend for eight to 12 hours. Nifedipine does not appear to interact with other drugs and has been safely used with beta-blockers, nitrates, diuretics, digoxin, anticoagulants, and other antihypertensive drugs.

The usual starting dose is $10 \mathrm{mg}$ three times daily $(5 \mathrm{mg}$ in the elderly). The recent introduction of a sustained release $(20 \mathrm{mg})$ preparation makes twice daily dosage possible in many cases. Dosage is increased until symptoms are relieved (or side effects occur) to a maximum of $120 \mathrm{mg}$ daily. An immediate effect may be obtained by biting the $10 \mathrm{mg}$ capsule and retaining the contents in the mouth.

Adverse effects-The risk of heart failure with nifedipine is small, but some patients have experienced worsening angina or cerebral ischaemia (perhaps because of a vascular steal phenomenon). About $6 \%$ of patients develop headache and others experience nausea, flushing, and dizziness, which are occasionally severe enough to merit stopping treatment. Ankle oedema may result from the higher proximal capillary pressure consequent on dilatation of the resistance vessels and does not usually respond to diuretics. In hypertensive patients already receiving beta-blockade because of angina the addition of nifedipine may produce severe hypotension without improving the angina; in those circumstances the calcium antagonist should be stopped. A similar profound hypotensive effect is sometimes seen when nifedipine is combined with beta-blockade in the early days after myocardial infarction. Drug induced hypersensitivity with hepatitis may rarely occur, as may glucose intolerance.
VERAPAMIL

Although verapamil is well absorbed after oral administration, it undergoes extensive first pass metabolism in the liver with a bioavailability of only $10-20 \%$. This explains why, in common with other highly extracted drugs such as propranolol, in order to achieve comparable plasma concentrations the oral dose is five to 10 times greater than the intravenous dose. The metabolites may have some pharmacological activity and are excreted by the kidney. The elimination half life varies from three to seven hours after a single dose, but during chronic dosing there is a fall in the first pass effect and the drug accumulates with a longer half life of up to 10 hours. Elimination is reduced in patients with liver disease. Verapamil is $90 \%$ bound to plasma proteins.

After intravenous administration the hypotensive effect is shortlived (10-20 minutes), but the depressant effect on the atrioventricular node persists for up to six hours, possibly because of preferential binding of drug to this tissue. The intravenous dose ( $5 \mathrm{mg}$ by slow injection over a minute) may be repeated after 10 minutes with blood pressure and electrocardiographic monitoring because hypotension and asystole have occasionally been reported in patients with myocardial damage. When given by mouth the dosage may, if necessary, be increased rapidly from 40 to $120 \mathrm{mg}$ three times daily over two to three days.

Adverse effects-The overall tolerance to verapamil is good. Many of the side effects-gastrointestinal disturbances (particularly constipation), headache and dizziness, and rashesmay be transient. Large doses may precipitate heart failure, but bradycardia, hypotension, and conduction disturbances are rare except when verapamil is given intravenously to patients receiving beta-blockers. In addition to a pharmacodynamic interaction with digoxin (see precautions) verapamil reduces both the renal and non-renal excretion of digoxin (similar to the interaction of digoxin and quinidine), thereby raising steady state digoxin concentrations by about $50 \%$.

\section{LIDOFLAZINE}

The precise mode of action and pharmacology of lidoflazine are not known. It appears, however, to act predominantly on myocardial and arterial smooth muscle without any major effect on myocardial contractility or conduction. Some studies have noted a small reduction in blood pressure and heart rate, particularly in response to exercise. Lidoflazine appears to be metabolised in the liver with an elimination half life of one to two days. In man, however, it often takes some weeks or months before the full clinical response is seen, and effects may continue for some time after treatment is stopped, which may indicate cellular accumulation of lidoflazine. This lack of immediate response greatly limits the usefulness of lidoflazine, especially when a quick benefit is desired.

Adverse effects-Gastrointestinal disturbances, dizziness, headache, or tinnitus occur in about $5 \%$ of patients at the outset of treatment, but these often disappear during long term treatment. Lidoflazine may cause prolongation of the Q-T interval in the electrocardiogram in some patients and may precipitate ventricular tachycardia especially in those receiving concurrent beta-blockade or in those taking antiarrhythmic drugs, a cardiac glycoside, or a potassium depleting diuretic. Rarely, disturbing dreams or hallucinations occur.

\section{PERHEXILINE}

Perhexiline is rapidly absorbed and extensively metabolised in the liver with renal and faecal excretion of metabolites. It is contraindicated in patients with severe liver or kidney disease. It is highly protein bound and has a long elimination half life of about two to five days. Possibly because of a genetic deficiency in hepatic hydroxylation, however, some patients metabolise the 
drug even more slowly with resultant pronounced accumulation and toxicity. Perhexiline, in addition to calcium antagonistic activity, has quinidine type activity and is a weak diuretic. It also acts by reducing the heart rate on exercise. It does not, however, appear to precipitate heart failure or airways obstruction.

Adverse effects-Perhexiline now has limited use because of troublesome and commonly dose related side effects in addition to those associated with calcium antagonists in general. Headache, dizziness, or nausea occur in about $30 \%$ of patients. Neurological side effects include tremor, incoordination, peripheral neuropathy, myopathy, papilloedema, and cerebellar and extrapyramidal syndromes. Weight loss is common and often severe. A mild rise in liver enzymes is common but usually reversible after discontinuation of treatment. Hypoglycaemia has also been reported. Perhexiline should not be prescribed unless the patient can be closely monitored (hepatic function, blood sugar, weight, eye examinations, etc) throughout treatment.

\section{PRENYLAMINE}

After rapid absorption prenylamine is metabolised by the liver and excreted both in the urine and the faeces. Again, caution must be exercised in patients with severe renal or liver disease. The elimination half life is about seven hours. Prenylamine also inhibits both uptake of catecholamine and release from storage granules, which may explain the absence of compensatory tachycardia in the presence of vasodilatation.

Adverse effects-Prenylamine mildly depresses myocardial contractility, lowers the heart rate both at rest and on exercise, and may precipitate heart failure. Large doses are associated with a tranquillising effect, and mild sedation and diarrhoea and rashes occasionally occur. Arrhythmias (especially in the presence of hypokalaemia) such as ventricular tachycardia and fibrillation (including a characteristic torsade de pointes) are the most serious and worrysome side effects. Prenylamine should be avoided when there is a risk of hypokalaemia or evidence of cardiac conduction defects.

\section{Uses of calcium antagonists}

\section{ANGINA}

Calcium antagonists may lower the oxygen requirements of the ischaemic myocardium by reducing myocardial contractility and by reducing blood pressure and therefore left ventricular pressure. In addition, they lower myocardial wall tension as a consequence of decreasing venous tone and therefore venous return. Unlike beta-blockers, they do not greatly reduce the heart rate. In those patients who develop spontaneous episodes of chest pain at rest or at night associated with reversible ST segment elevation in the electrocardiogram (variant and Prinzmetal's angina) and thought to be associated with coronary artery spasm (vasospastic angina), calcium antagonists such as nifedipine, which particularly affect vascular smooth muscle and thus relieve spasm, effect dramatic relief in about $80 \%$ of patients. Reports suggest that if patients become asymptomatic and remain so after a few months the drug may be cautiously withdrawn. Calcium antagonists are also synergistic in combination with beta-blockade in many patients with classic exercise induced angina, when presumably some degree of coronary spasm accompanies structural coronary artery stenosis. Given alone, they are generally less potent than beta-blockers in preventing classical angina. Nevertheless, they may be very useful when beta-blockers are contraindicated.

\section{SUPRAVENTRICULAR TACHYCARDIA}

Many supraventricular tachycardias, including atrial flutter and those associated with the Wolff-Parkinson-White syndrome, are maintained by a re-entry phenomenon using the atrioventricular node. Those calcium antagonists that have a prominent influence on the atrioventricular node-for instance, verapamilreduce the fast ventricular response to these arrhythmias and to atrial fibrillation and may also sometimes prevent or abolish them (see article on antiarrhythmic drugs). Prolonged atrioventricular conduction induced by excess verapamil can best be reversed with atropine. Nifedipine does' not have antiarrhythmic properties, and calcium antagonists are not helpful in managing ventricular arrhythmias.

\section{HYPERTENSION}

Calcium antagonists relax arterial muscle and so reduce raised blood pressure, though nifedipine, because of its pronounced effect in lowering smooth muscle tone, usually has a more potent antihypertensive effect than verapamil. This hypotensive effect is more striking when nifedipine is prescribed in combination with beta-blockade. Postural hypotension and tachycardia may result from its use. Nifedipine has also been used successfully in combination with methyldopa in the long term treatment of severe hypertension. The hypotensive potency of nifedipine is similar to that of hydralazine, and that of verapamil is similar to that of thiazide diuretics. The full potential of these agents in hypertension has not yet been fully explored. At present they are not recommended for first line treatment but may be used in combination with other antihypertensive drugs, particularly when a vasodilator is indicated.

\section{OTHER INDICATIONS}

The use of calcium antagonists in chronic heart failure, hypertrophic cardiomyopathy, and pulmonary hypertension and to preserve the myocardium during acute infarction is currently restricted to investigative work. Preliminary observations suggest that nifedipine may produce symptomatic relief in patients with Raynaud's syndrome.

\section{General precautions}

Most calcium antagonists have a negative inotropic effect and so may induce heart failure, particularly when used in high doses and when the sympathetic drive to the heart is blocked by concurrent beta-blockade. Special care should be taken in elderly patients. Remember that, should the drug induced reduction in myocardial contractility produce cardiac dilatation, the oxygen demands of the failing heart may be increased and angina exacerbated. Difficulties with atrioventricular node conduction may result in heart block especially when a calcium antagonist drug, and particularly verapamil, is used in combination with another drug that prolongs atrioventricular node conduction-for example, digoxin, beta-blockers, and some antiarrhythmic drugs. The combination of beta-blockade and verapamil, particularly when verapamil is given intravenously, may produce heart block (and asystole) in some patients and should be avoided. The oral combined use of nifedipine or verapamil and a beta-blocker has been safely used in angina, but some authorities still caution against the use of even oral verapamil with a beta-blocker, recommending a 24 hour interval after withdrawal of the beta-blocker before verapamil is started. Hypotension, with consequent serious myocardial underperfusion and syncope, may result from the combined negative inotropic effect, and severe arterial and venous dilatation may result when the usual compensatory increase in stroke volume is prevented-for instance, in those with aortic stenosis or a fixed obstruction to left ventricular ejection or receiving concurrent beta-blockade or with raised ventricular diastolic pressures. Calcium antagonists should therefore be used cautiously in those receiving other hypotensive drugs. 


\section{New nitrates}

It is now thought that nitrates act primarily by relaxing vascular and particularly venous smooth muscle, thus decreasing the return of blood to the heart. They therefore reduce the demand for myocardial oxygen by decreasing left ventricular volume, filling pressure, and to a lesser extent also afterload. They probably increase the supply of oxygen by causing redistribution of coronary blood flow (dilatation occurs only in normal vessels) and increasing collateral flow, particularly to ischaemic regions. The nitrates are primarily used in angina pectoris, and several new preparations have become available. The recent use of nitrates in acute myocardial infarction and congestive heart failure, although producing favourable haemodynamic response, is to date too limited to recommend their widespread use in these conditions.

Sublingual glyceryl trinitrate (nitroglycerine, trinitrin) is rapidly absorbed and is the most widely used drug in treating angina because of rapid absorption and almost instantaneous achievement of high serum drug concentrations. The drug has recently also become available as a metered dose aerosol that delivers $0.4 \mathrm{mg}$ glyceryl trinitrate per spray emission (Nitrolingual spray) on to the oral mucosa, especially under the tongue. Unlike sublingual tablets, which may rapidly lose potency (often indicated by a loss of characteristic tingling or burning sensation) if kept in a warm place or non-opaque bottle, this form of glyceryl trinitrate is chemically stable for at least three years. Its efficacy and adverse reactions (headache, flushing, and postural hypotension) are similar to those of the sublingual tablets. It is, however, considerably more expensive, but it may produce even more rapid relief from angina in some patients. Buccal glyceryl trinitrate (Suscard, buccal) is placed above the upper front teeth between the gum and lip. It has both an initial partial dissolution producing a rapid onset and a sustained effect for four to six hours that is quickly reversed by removal from the mouth should side effects occur. More information about its performance in patients with dentures and during eating and drinking is required. Experience to date with these two new formulations is limited.

Glyceryl trinitrate unfortunately has only a short acting effect (usually 20-30 minutes). Small doses of oral long acting nitrates have proved disappointing because of loss of systemically available drug due to "first pass" metabolism in the liver. Recent investigations have shown objectively by formally testing exercise capacity, ST segment depression in the electrocardiogram, and haemodynamic and biochemical monitoring that large doses of oral nitrates-for instance, isosorbide dinitrate $(5-20 \mathrm{mg}$, two to four times daily) or sustained release glyceryl trinitrate-are effective, presumably because the doses are sufficiently large to at least partially offset first pass metabolism. Nevertheless, isosorbide dinitrate is subject to a pronounced and highly variable first pass metabolism to 2- and particularly 5-mononitrates, both of which are haemodynamically active. The 5 -mononitrate of isosorbide has recently become available (Elantan, ISMO-20), and higher and more consistent plasma nitrate concentrations may be obtained with this drug than with an equal dose of sustained release isosorbide dinitrate. In reducing anginal attacks consumption of glyceryl trinitrate and increasing exercise tolerance to date both appear equally efficacious. Mononitrate is rapidly absorbed and is excreted by the kidney both unchanged and also as an inactive glucouronide metabolite, with an elimination half life of between four and six hours. Its clinical efficacy correlates with its plasma concentration, being about eight hours for a 20 $\mathrm{mg}$ dose. Dosage is $20-40 \mathrm{mg}$ three times daily. Whether this form of isosorbide will prove more effective clinically than other isosorbides remains to be established. Nevertheless, the effectiveness of these longer acting nitrates is no longer in doubt.

Nitroglycerine is also absorbed from the skin but much more slowly than from the sublingual mucosa, again bypassing the liver. Numerous studies have shown a prophylactic antianginal effect of glyceryl trinitrate ointment (Percutol). The degree and duration of the benefit are directly related to the amount of ointment applied. Normally $1 \cdot 2-5 \mathrm{~cm}$ (about $8-32 \mathrm{mg}$ ) of ointment is measured on to a special sheet (Applirule) and applied to the skin, without being rubbed in, and taped into position. The dose applied may be increased to just below that which produces troublesome nitrate induced headaches, but individual patients are best advised to find their own most suitable dose and times of application as there is considerable patient to patient variation. The effects last four to six hours or sometimes longer, irrespective of where on the skin the ointment is applied. For added psychological benefit it is often applied to the chest wall. A self adhesive patch impregnated with glyceryl trinitrate (Transderm-Nitro) that is released slowly over 24 hours is also available for prophylactic use. It resembles a sticking plaster, and effective plasma concentrations are usually achieved within two hours of application. Skin irritation may occasionally occur but is less likely if the site of application is varied.

Glyceryl trinitrate ointment has proved particularly useful in those with atypical or variant angina at rest, after exercise, or causing the patient to waken during the night, when, as mentioned above, it is often associated with coronary spasm. In these cases the ointment has been shown to abolish the ST segment elevation as well as the pain. It has also helped to reduce use of sublingual nitrate in exercise induced angina.

Adverse effects common to all nitrates are related to vasodilatation-throbbing headache, flushing, postural hypotension, and reflex tachycardia-and may be minimised by removing the source of nitrate-for example, spitting out sublingual glyceryltrinitrate after angina has been relieved or removing patches or ointment. Although some patients may find these effects too troublesome to persist with treatment, tolerance to them commonly occurs during prolonged treatment.

\section{Bibliography}

Anastassiades CJ. Nifedipine and beta-blocker drugs. Br Med $\mathcal{F} 1980 ; 281$ : 1251-2.

Heart failure induced by nifedipine.

Anonymous. Calcium antagonists and the heart. Br Med $\mathcal{f} 1981$;282:89-90. General review of the calcium antagonists and their clinical role.

Anonymous. Calcium antagonists in hypertension. Lancet 1982 ;ii:307-8. General review of their increasing use and possible mode of action in hypertension.

Subramanian VB, Lahiri A, Paramasivan R, Raftery EB. Verapamil in chronic stable angina. Lancet $1980 ; \mathrm{i}: 841-4$.

A controlled study with computerised multistage treadmill exercise.

Bowles MJ, Subramanian VB, Davies AB, Raftery EB. Comparison of antianginal actions of verapamil and propranolol. $\mathrm{Br}$ Med $\mathcal{F} 1981 ; \mathbf{2 8 2}$ : 1754.

A help for which drug to choose first for whom.

Braunwald E. Mechanism of action of calcium-channel-blocking agents. $N$ Engl f Med 1982;307:1618-27.

The role of calcium and the effects of these agents on the cardiovascular system is considered in detail. The expanding therapeutic use of calcium antagonists is outlined.

Dean S, Kendall MJ. Adverse interaction between nifedipine and betablockade. $B r$ Med f 1981;282:1322.

Eklayam U, Aronow WS. Glyceryl trinitrate (nitroglycerin ointment and isosorbide dinitrate). Drugs $1982 ; 23: 165-94$

A review of the pharmacology and therapeutic use of nitrates.

Kahan A, Weber S, Amor B, et al. Nifedipine for Raynaud's phenomenon. Lancet $1983 ; \mathrm{i}: 131$.

A double blind study reporting symptomatic relief; allied correspondence (pp 130-1) emphasises the need for objective evidence.

Lynch P, Dargie H, Krikler S, Krikler D. Objective assessment of antianginal treatment: a double-blind comparison of propranolol, nifedipine and their combination. $\mathrm{Br} M e d F 1980 ; 281: 184-7$.

Shows that the combination is better than either drug alone.

Nobile-Orazio E, Sterzi R. Cerebral ischaemia after nifedipine treatment. Br Med F $1981 ; 283: 948$

Olivaria MT, Bartorelli C, Polese A, Fiorentini C, Moruzzi P, Guazzi MD. Treatment of hypertension with nifedipine, a calcium antagonist agent. Circulation 1979;59:1956-62.

Zelis R, Flaim SF. Calcium blocking drugs for angina pectoris. Ann Rev Med $1982 ; 33: 465-78$.

A consideration of the manifestations of coronary vasospasm and a review of the current experience with verapamil, nifedipine, and diltiazem. (The latter is not yet available in the UK.) 